\title{
Mak Yong Music of Malaysia: Negotiating Complex Musical Content Within Periodicity
}

Patricia Matusky, Cultural Centre, University of Malaya, Kuala Lumpur

pmatusky@gmail.com

(C) 2015 University of Malaya. All rights reserved.

Malaysian Journal of Performing and Visual Arts, Volume 1, 2015

\begin{abstract}
Over the past many centuries the dance theater known as the mak yong has been a major form of indigenous Malay theater that relates stories using formal and improvised monologue and dialogue, stylized dance and acting, and vocal as well as instrumental music. While written historical records about any aspect of the musical style prior to the late- $19^{\text {th }}$ century do not exist, the musical features of the mak yong since the early to mid- $20^{\text {th }}$ century are evident to us today through remembered and live performances by mak yong artists who are still active in the 21 st century. This article explores the musical style of the mak yong, dating from the early $20^{\text {th }}$ century to current times, noting the musical features of periodic formal structures, resultant rhythms and a complex melodic content that bind this genre to aspects of an ancient Southeast Asian musical style as well as to influences from Middle Eastern musicculture.
\end{abstract}

Keywords: mak yong, makyung, periodicity, Malay traditional theatre, Malay traditional music, melodic ornamentation, interlocking performance style, rebab, Southeast Asian theatre

\section{Introduction}

In the ancient dance drama known as mak yong, originating among the Malay people in the region of northeast Malaysia (Kelantan) and southern Thailand (Patani and Narathiwat), exists a folk theatrical that is rich in story, dance and musical content. This theatrical uses formal and improvised monologue and dialogue, stylized dance, acting, and vocal and instrumental music. ${ }^{1}$ Thought to have originated in the mainly Malay-dominated rural areas of the coastal states of Kelantan (Malaysia) and Patani and Narathiwat (south Thailand), this theatrical flourished in the late $19^{\text {th }}$ and throughout most of the $20^{\text {th }}$ centuries in Kelantan, but today it is rarely performed in its homeland state. ${ }^{2}$ Formerly, the distribution of the mak yong village style was wide, found in the states of Kelantan and Kedah (north Malaysia), Patani and Narathiwat (south Thailand), in northeast coastal Sumatra, and southward to the Riau Islands of Indonesia where a variant of the mak yong is still performed today.

Ancient myths and legends as well as its connection to the main puteri healing ritual of Kelantan tie this theatrical to ancient times and to a flourishing period of late- $19^{\text {th }}$ and $20^{\text {th }}$ century performance (Ghulam Sarwar 1976). Today, a village performance of mak yong is formally prohibited in its home state of Kelantan, however, it is taught in the urban areas of the country, notably at the National Academy of Arts, Culture and Heritage (ASWARA). Occasional performances also occur in the National Theatre in Kuala Lumpur which tend to feature productions that are eclectic in stage design, costuming and dance styles, and which also exhibit rehearsed and glamorized performances as well as shorter overall performance times that cater to the urban audience. There are some indications of questionable sustainability today, in spite of a very rich and complex musical style, use of distinctive and elaborate costumes, as well as highly stylized and sophisticated dance styles emanating from a village folk tradition. 
Early documentation and analysis of performance structure of the mak yong in Kelantan was carried out in the early 1970s (Mohd. Affandi Ismail 1973-74, 1975; Ghulam Sarwar Yousof 1976, 1982, 1992 and so on); these studies and subsequent publications have focused mainly on the theatrical aspects and the oral literature of this theatrical genre. In contrast, studies on the musical style of the mak yong are few, with early publications based on preliminary research in the late 1960s (Malm, 1969, 1971, 1974) and some 10 years later by the present author; however, more focused efforts occurred some 20 years later in a study for a Master's degree by the Malaysian composer Sunetra Fernando (Fernando 1996) in which a single piece, known as Menghadap Rebab, a ritual song cum dance piece, was the focus in a work on musical analysis, function and meaning of the given piece in the context of Kelantanese culture.

In an effort to enrich the musical literature on the mak yong theatre of Kelantan, this article explores the musical style of the Malaysian mak yong of the $20^{\text {th }}$ and $21^{\text {st }}$ centuries, arguing that musical formal structures are governed by rigid periodic (time) units that govern the occurrence and placement of supporting rhythmic patterns as well as a highly complex melodic content. In effect, the complexity of melodic content along with lyrics, most of which are improvised, are in constant negotiation with the elements of the musical structure dictated by iso-periodic units that are repeated within a given piece, and which secure a firm framework for all other elements in the music.

The following analysis will show that at times the complex melodic and lyric content takes a dominant role in the musical structure, dictating tempo, the occurrence of drum timbres, rhythmic patterns, accented patterns and other musical elements, while at other times the periodic structures are the dominating elements that provide an inflexible and firm basis for tempo, resultant rhythmic patterns and melody that are rendered by vocal parts as well as instrumental music that often accompanies dance steps. This argument is supported through analysis and discussion of musical elements that focus on instrumentation, characteristics of musical form that are rooted in periodic formal structures, rhythmic patterns that are dependent on interlocking performance styles, and melodic content characterized by a high degree of ornamentation as well as heterophonic relationships in texture between voice, instrument and group vocal singing.

The periodic formal structures and the complex melodic elements in the music, and the negotiation that occurs between them in order to create beautiful melodic lines in the various pieces of the repertory, find their origins in a musical tradition with strong ties to indigenous musical elements of 'island' Southeast Asia. At the same time, also evident are influences from mainland Southeast Asia and also from Middle Eastern musical traits associated with Islamic culture arriving on the Malay Peninsula in times long ago by traders and settlers from Middle Eastern countries.

\section{Outline of Theatre Conventions, Role Types, Dramatic and Musical Repertory}

Traditionally in the village, mak yong is performed at ground level in a performance area that is openwalled and covered by a thatched roof structure. The ground area is usually covered by woven mats (tikar) on which both the musicians and the actors and actresses sit and perform. In a town or urban setting the performance may take place on a raised and roof-covered platform sometimes with a plain backdrop on one side of the stage (see Figure 1). ${ }^{3}$ 


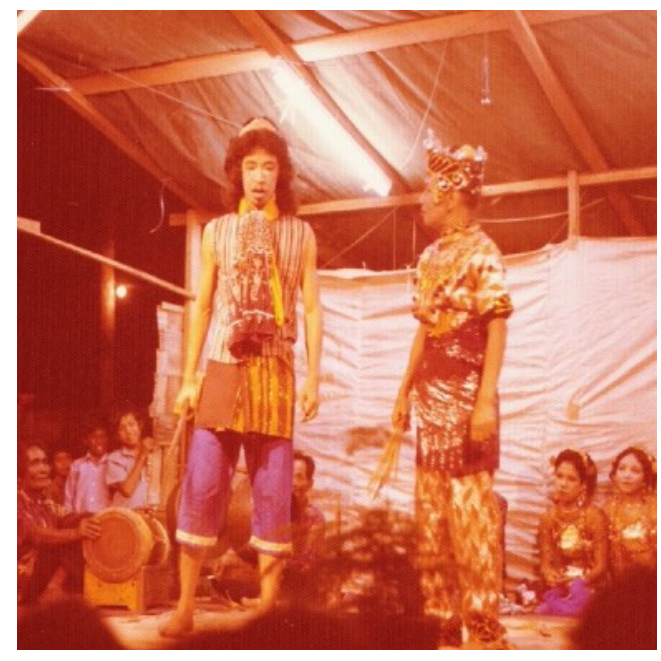

Figure 1. Mak yong performance on a raised platform stage with roof, open for viewing on three sides.

(Performance in 1976, photo by H. Yamaguchi.)

The dramatic repertory of the mak yong comprises twelve Malay folk tales with themes related to the adventures of culture heroes, with the original and principal story entitled 'Dewa Muda'. Many of the eleven additional stories may have developed from the principal story and some may even have been borrowed from other theatrical genres of the geographic region, genres such as menora, wayang kulit and the bangsawan (Ghulam Sarwar 1976:83-90). Adventures of kings and princes are featured in most stories that may also include supernatural events.

All characters are portrayed by specific role types such as the Pak Yong and Pak Yong Muda (king and prince, respectively, who are the leading male characters played by women since the early $20^{\text {th }}$ century), the Mak Yong and Puteri Mak Yong (queen and princess, leading female characters), the Peran Tua and Peran Muda (the old and young clown-servant characters, played by males, see Peran Muda in Figure 1 above), the Inang (female attendant), the Tuk Wak (an astrologer or expert of some kind, played by a male), and other roles types such as jin and gergasi (spirits and ogres), orang darat (country folk) and various birds and animals. Very few props and no stage sets are used in traditional performances, and places and times in a given story are all told through speech or song.

The first night of a performance is marked by specific events. A bomoh (Malay shaman) is invited to officially open the stage (buka panggung), to read the kenduri (prayers) and to execute all ceremonies to properly open the stage for performance. In the course of events the bomoh often sings the greeting song (bertabik) using the musical piece (lagu) entitled 'Sedayung Pak Yong' to welcome the performers and audience. After the opening, the small music ensemble plays an opening musical prelude, and eventually the entrance of the actresses is signaled by the instrumental piece entitled 'Pak Yong Turun' (the Pak Yong descends'). Following are a standard number of musical and dance pieces beginning with an elaborate number known as 'Menghadap Rebab' (song and dance to pay homage to the rebab bowed-lute). After the opening number, in sequence, are additional pieces such as those entitled 'Sedayung Mak Yong' and 'Sedayung Pak Yong' (preparatory songs for the respective character types), the piece entitled 'Ela' (sung by the Pak Yong for character self-identification); finally, the story for the evening begins (Ghulam Sarwar 1976: 164-181).

The musical repertory of the mak yong theatre numbers around thirty different pieces comprising both purely instrumental pieces (referred to as lagu palu-paluan, 'drummed pieces') and songs (lagu nyanyian, 'sung pieces'). ${ }^{4}$ The drummed pieces accompany specific activities in the performance such as the 
entrance of the actresses at the beginning of a show, the closing of a performance, and also the action of traveling or quick scene and other changes within a story.

On the other hand, most pieces in the repertory are sung by a soloist (actor) and a small chorus. Vocal music comprises many different types of songs used in specific situations in the performance itself. Some examples are the piece known as 'Menghadap Rebab' (the salutation to the rebab bowed-lute which is the ritual dance and song at the beginning of a performance), the pieces for relating news of some kind (called berkabar or cerita bari), pieces for lullaby and other intimate situations in a story, pieces for walking or travelling such as the titles 'Timang Welu' and 'Kijang Mas', pieces for lamenting (lagu 'Mengambul'), and songs for special activities as they arise in the drama.

A special category of song is referred to as 'sedayung' - there are at least eight different titles of 'sedayung', and each one is characterised by a particular dramatic situation and musical characteristics. The 'Sedayung Mak yong', for example, is sung in the opening prologue and also many times during a performance by the Mak Yong character for various purposes (to express an idea, to reflect on a situation and so on).

In contrast, special circumstances are often attached to the song 'Sedayung Pak Yong' (usually sung by the Pak Yong character). This piece carries strong association with power (sakti) and is used in situations of transformation or to invoke special powers in the drama at hand (Ghulam Sarwar 1976). Noting the frequent use of this 'sedayung' piece in performance and its overall importance in many different dramatic situations, it will be used in this article to exemplify a typical mak yong musical soundscape and to serve as the central point and discussion of this article which focuses on musical aspects in this traditional folk theatrical.

\section{The Music Ensemble}

A small percussion-dominated ensemble provides the musical accompaniment for the mak yong, and occasionally additional instruments are borrowed, as needed, from other neighboring theatricals such as the wayang kulit Kelantan and the menora of south Thailand. This small orchestra, emphasizing percussion instruments (idiophones and membranophones) along with a single melody instrument (the rebab spiked fiddle chordophone), is consistent with many other theatrical ensembles in Malaysia and, indeed, in Southeast Asia.

The main instruments in the mak yong ensemble are two tetawak (or tawak) large hanging knobbed bronze gongs, two gendang hand-hit drums, and one rebab bowed-lute - a spiked fiddle (see Figure 2).

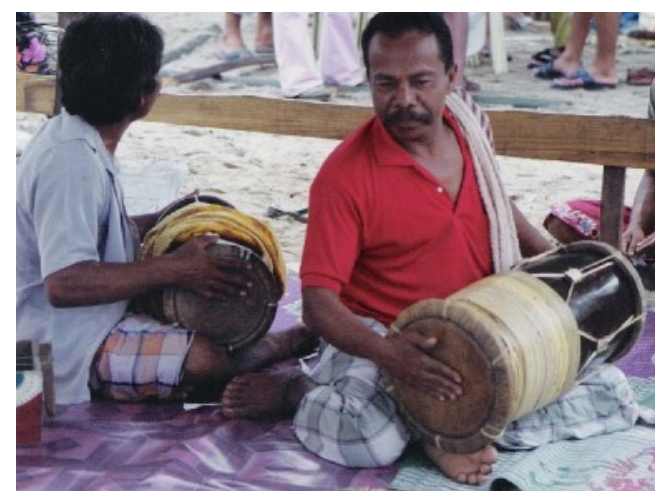

Gendang

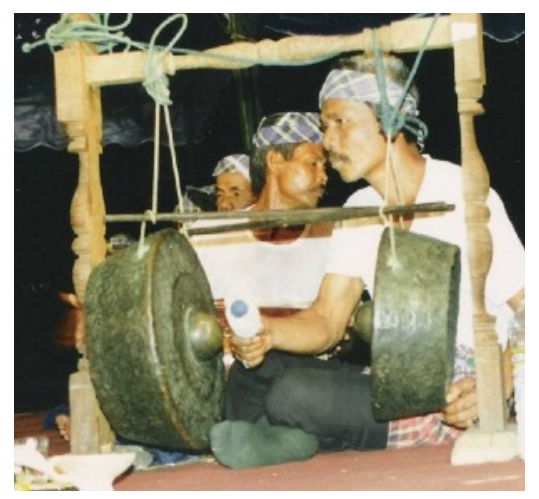

Tetawak 


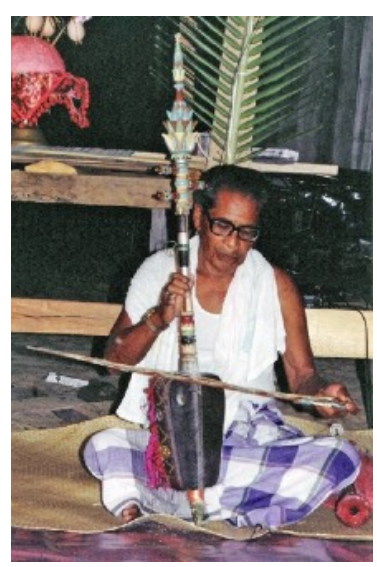

Rebab

Figure 2. The main musical instruments in the makyong theatre.

The main melody producing instrument, aside from the human voice, is the rebab, a 3-stringed bowedlute which is most likely derived from Middle Eastern sources, although it appears in a form and style that is unique to Malaysia. The long fretless neck, slightly elongated and triangular-shaped body, the elaborately carved and decorated head piece (kecopong), along with the use of three strings and lateral tuning pegs distinguish this rebab from others in the Middle Eastern and Southeast Asian worlds. The three strings are supported by a small moveable bridge located near the top of the front of the body, and a small lump of beeswax is attached to the body near the bridge to slightly mute the sound. The strings are bowed with an intricately carved wooden bow with nylon string attached (these replace the coconut fibre strings used in the past) which are usually coated with resin to enable smooth bowing on the metal strings of the instrument as the player's hand tautens the bow strings. The rebab player provides highly ornamented melodic lines in a heterophonic relationship with the singer's voice.

The percussive rhythmic patterns in the music are provided by two gendang drums, in large and small sizes (often referred to as gendang ibu [lit., 'mother' gendang], and gendang anak ['child' gendang]), that are played in an interlocking style by two players to produce resultant rhythmic patterns. As the drums are hand-hit, the players use specific timbres of sound on each of the drumheads to produce specific rhythmic patterns that accompany the singing and that are associated with specific pieces (Matusky 1980, 1994). The drummers also determine the tempo of certain pieces, whether sung or instrumental, and provide the sense of a running beat in the overall musical texture.

Finally, the large hanging knobbed bronze gongs, called tetawak or tawak, are hung on ropes from a rack or from the roof beams of the mak yong stage. The two gongs provide a high pitch and low pitch when hit on the knob with a padded beater; ideally these two pitches are approximately an interval of a third or as large as a fifth apart. Played by one musician, this set of idiophones provides the periodic structure (or gong unit) that serves as the basis of musical form for a song or instrumental piece. The tetawak gongs have very thick walls, a deep rim and a rough dark surface with a knob on the center face of the gong, and they exemplify the kind of knobbed gongs found throughout the Malay Archipelago including Borneo, the islands of Indonesia and the southern Philippines.

Other instruments that may be borrowed from other theatricals from time to time include the serunai freebeating reed aerophone, the gedumbak goblet-shaped drum or the short barrel geduk drum that is hit with a pair of drum sticks. These instruments are used for particular dance pieces or other special tunes as needed in a given performance. ${ }^{5}$ 


\section{The Music}

The polyphonic music found in the makyong theatrical is particularly characterized by a stratified texture of melody, percussive rhythmic patterns and periodic gong units. As noted earlier, very complex melodic lines are rendered usually within the context and the structure of very rigid and exact musical forms as provided by the tetawak gongs in the form of periodic structures. As further conversationalists in this musical soundscape, the drum rhythmic patterns dictate tempo and reveal stressed beats and patterns, running beats in the music and other aspects of musical content. As noted earlier, the analysis and observations that follow in this discussion are based on two types of the piece known as 'sedayung' (meaning unknown), that is, the pieces 'Sedayung Mak Yong' and 'Sedayung Pak Yong', both of which are heard frequently in a mak yong performance and both of which exhibit clear characteristics that are commonly found in mak yong pieces in general.

Musical Form The musical form or structure in mak yong pieces is predicated on a repeated periodic (or time) unit that may be referred to as a 'gong unit' since, in all pieces, it is rendered by the two large hanging tetawak gongs (Matusky 1994, 1997, 2012). The concept of periodicity (Tenzer 2006) encompasses units of musical time which may be cyclical or metric in nature, each type with a specific set of characteristics. Periodic units specify musical form in a piece and are usually unchanging, whether they are cyclic or metric types. However, in some musics the periods can undergo processes of change of some kind, in which case they may be described as being 'discursive' (Tenzer 2006). In this case, the given discursive periodic unit may become less important in specifying musical form, although still present in the music, while other elements in the musical texture assume the dominant role in determining form.

In the mak yong musical tradition the periodic units are cyclical in nature and are infinitely repeatable, therefore the periods may be further referred to as iso-periods (that is, 'same' period) that are successively repeat over and over in a given piece of music. In mak yong and other traditional Malay musics in which form is determined by iso-periodic structures, such as the wayang kulit, joget gamelan and others, the cyclic period is also binary in nature in that any given gong unit contains a number of beats that is a multiple of two; hence, gong units in mak yong music are 8-, 16-, 32-, 64-beats, and so on in length. Furthermore, gong units feature hierarchical beats in that some beats are more important and carry greater stress than others in a given gong unit. In general, gong units are end-accented and within any given gong unit a 2-level stress unit (a beat of weak stress followed by a beat of strong stress) is in operation and repeated in order to comprise the total number of beats in a given periodic unit. Finally, all gong units are concatenated, they are chained together at the occurrence of the final beat of the gong unit (signified by the sounding of the low-pitched tetawak) which becomes the beginning of the next successive gong unit or iso-period (Becker 1980).

While the low-pitched tetawak sounds only on the final beat of a given gong unit, the high-pitched tetawak marks internal beats within the gong unit by sounding on specific beats that are determined by the subdivision of the gong unit in a binary way (Matusky 1980, 1994 and Becker 1979, 1980). The isoperiod (or gong unit) is an important aspect in the performance of mak yong music (and in the music of other traditional theatricals), for it not only gives form to the musical sound, but the punctuating gong tones also act as signaling devices for the singers, dancers and chorus as noted in the discussion that follows.

The musical form for of the piece 'Sedayung Mak Yong' is based on a cyclic iso-period (or gong unit) of 32 basic beats as shown in Figure 3. In this gong unit, the final beat (beat 32) is always marked by the low-pitched tetawak. This 32-beat gong unit is subdivided internally by the sounding of the high-pitched tetawak which is played on beats 8,16, 24 and 28 (subdividing the gong unit into quarter-segments, and a single eighth-segment near the end of the gong unit). Additionally, the high tetawak pitch is played on beats 28 and 30, which serve as a signaling device for the approaching end of the gong unit and the sound of the low-pitched tetawak on beat 32 (signifying the end of the given unit and the beginning of the next successive gong unit). These occurrences of gong tones and the iso-periodic structure is shown in Figure 3 in standard music notation. 


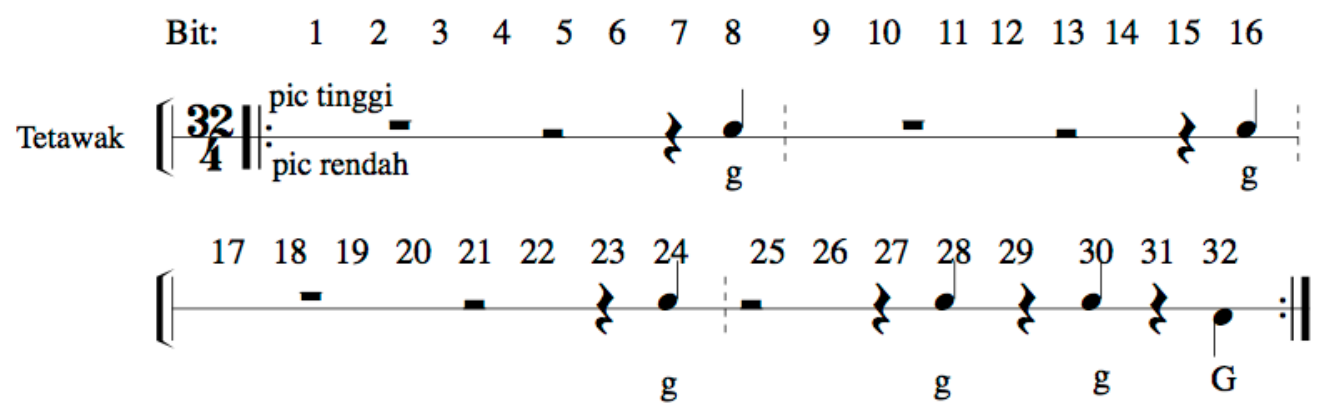

Figure 3. Cyclic iso-period (gong unit) for the song 'Sedayung Mak Yong', in standard music notation showing high pitch [pic tinggi] and low pitch [pic rendah].

In actual performance, all mak yong pieces actually begin by the drummers (and the gong player) on a specific beat within (usually near the middle of) the given gong unit and proceed to the end of the unit; it is only at the sound of the low-pitched tetawak that the end of the gong unit is realized, and from that point onward the complete unit is heard in successive repetitions throughout the given piece. The dual role of the low-pitched tetawak, as (a) marker of the end of one gong unit and simultaneously (b) the marker of the beginning of the successive repetition of the gong unit, informs us that the iso-period (gong unit) is, indeed, a cyclic event in this music system.

The piece "Sedayung Pak Yong" is also cast in a cyclic iso-period (gong unit), however, it is 16 beats in length. The internal gong marker high-pitched tetawak gong is played on beats 4,8,12, and 14, in effect subdividing the gong unit in the same configuration as the piece 'Sedayung Mak yong' (into quartersegments, and then into one eighth-segment). The low-pitched tetawak gong is played only on the final beat 16 of this repeated iso-periodic unit and it, along with the high-pitched gong tones, provide the musical form for the piece 'Sedayung Pak Yong'. The 16-beat gong unit is shown using standard music notation in Figure 4 below.

Bit: $\quad \begin{array}{lllllllllllll}1 & 2 & 3 & 4 & 5 & 6 & 7 & 8 & 910 & 11 & 121314 & 1516\end{array}$

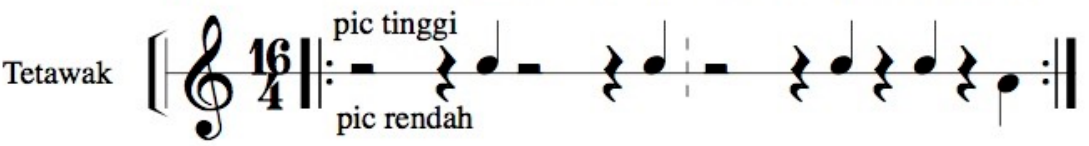

Figure 4. Cyclic iso-period (gong unit) for the piece 'Sedayung Pak Yong'.

The periodic structures (or gong units) of both 'Sedayung' pieces noted above are very similar, and the piece 'Sedayung Mak Yong' maybe be seen as an expanded form of the piece 'Sedayung Pak Yong' (here we see the expansion of a 16-beat gong unit to a 32-beat unit), or alternately, the 'Sedayung Mak Yong' piece may be seen as a contraction of the 32-beat unit to a 16-beat gong unit as found in 'Sedayung Pak Yong'. In any case, the two forms are intimately related in terms of musical form and periodic structure, and both carry the basic characteristics of cyclic periods as noted above. While both periodic structures are extremely basic and simple in configuration and in sound content, both also are highly rigid and stable time units within the musical system. Although the periodic units appear to be minimal in musical sound, yet their presence in the musical system, and indeed in a given piece, provides a solid basis for all the other parts of the musical content. In effect, in an analysis of the music, the periodic unit is usually the dominant part of the musical sound that provides a specific musical form in the music.

Rhythmic Patterns and Vocal Lines The two gendang drums provide the percussive rhythmic content of mak yong pieces. The percussive rhythmic patterns in this musical system are performed by two different drummers who play in an interlocking style to produce resultant rhythmic patterns with specific internal structure, and which are played within the confines of the periodic gong unit for the given piece. The 
drum patterns are rendered with specific mnemonic sounds played in specific locations on the hand-hit drumheads. These sounds are onomatopoetic drumming timbres that are vocalized by many drummers as the sounds 'chak', 'ting', and 'duh', and are used in Figures 5 and 6 to notate the specific resultant drum patterns discussed here.

The resultant drum patterns always occur within the context of the periodic gong unit, and their overall length and structure is synchronized with the length and structure of the given gong unit in which they occur. The rhythmic patterns comprise standard rhythmic formulae as well as the improvisational interplay between the two drummers from one gong unit to the next. The basic, unornamented drum patterns are usually 4- or 8-beats in length and comprise the standard formulas or building blocks that make up the complete given rhythmic pattern in a specific piece.

The piece 'Sedayung Pak Yong' is cast in a 16-beat iso-periodic time unit that is performed in a moderate tempo (about 50MM per crochet-note beat). Both the basic structure of the resultant rhythmic pattern and the improvisational, ornamented version of the basic pattern are always structured in the context of the 16-beat gong unit as noted above in Figure 4 and as shown in score in Figure 5. The internal structure of the resultant rhythm comprises two contrasting 4-beat phrases, each of which ends simultaneously with the sounding of the high-pitched tetawak gong tone at the end of the quarter-segment of the periodic time unit. The two phrases are noted as $a, b$ in the illustration below in Figure 5a, and repeated with some slight variation in drum timbres, noted as $a 1, b 1$ to complete the 16-beat gong unit (thus, the complete 16-best drumming pattern may be outlined as $a, b, a 1, b 1)$.

a. basic pattern

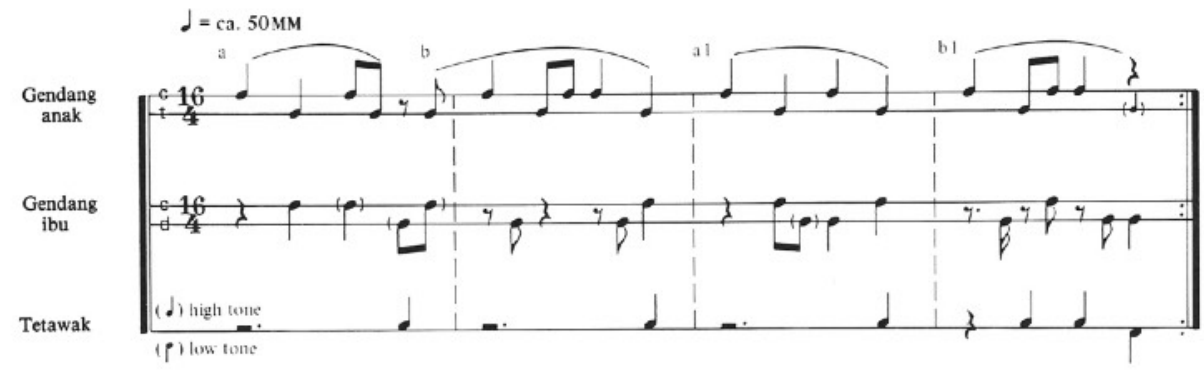

b. ornamented pattern

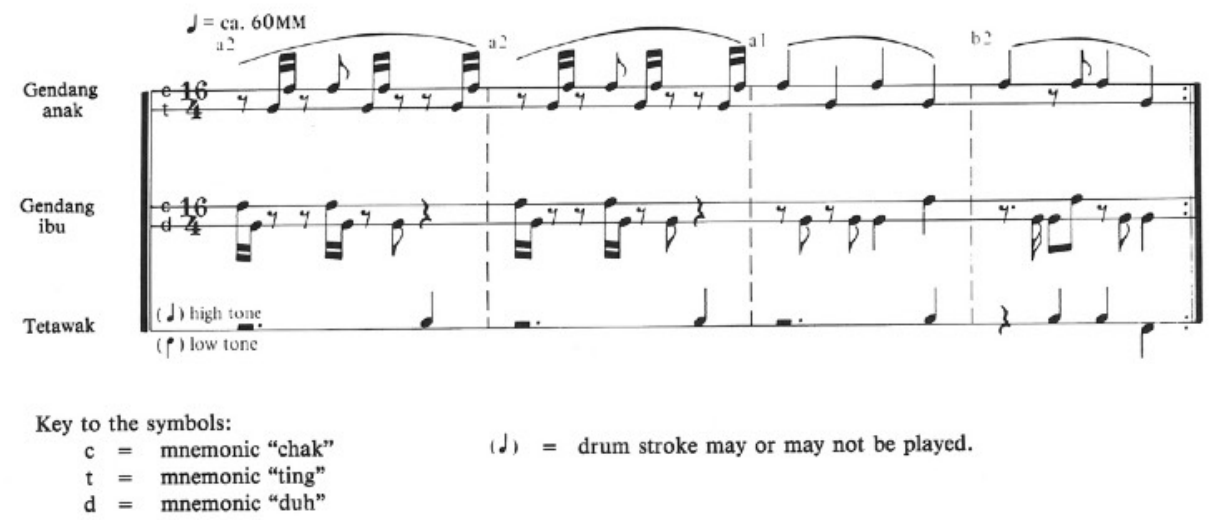

Figure 5. Resultant rhythmic patterns and the 16-beat cyclic iso-period for the piece 'Sedayung Pak Yong', showing a) basic pattern and b) ornamented pattern.

In the analysis of the song 'Sedayung Pak Yong" the cyclic iso-period is the part in the musical texture that determines the basic musical form of the song. The drumming patterns always conform to the 
specific length of the gong unit (the tetawak part in the score) and its stressed beats as shown above in Figure 5. When the vocal and rebab melodic parts are added to this conversation in the musical texture, the solo voice part is sung within the context of one complete 16-beat gong unit, and the following chorus part also conforms to the length of one complete 16-beat gong unit. The solo and chorus alternate their parts, following the structure set by the iso-periodic time unit.

As the piece 'Sedayung Pak Yong' progresses the tempo increases, the drummers begin to play improvised, ornamented versions of the basic 4-beat patterns as shown in Figure 5b. While the internal form of the resultant patterns takes a slightly different form (see Fig. 5b, drumming pattern outlined as $a 2, a 2, a 1, b 2$, an antecedent-consequent form), the improvisation still takes place within the 16-beat gong unit and the 4-beat phrase $b$ continues to serve as a cadential phrase in the drumming pattern, ending simultaneously with the low-pitched gong tone on beat 16 of the iso-periodic unit.

In contrast, the piece 'Sedayung Mak Yong' exhibits a more intense interaction of textural parts in the music and the conversation among the parts (that is, the gong unit, the drum rhythmic patterns and the melody) becomes more intricate and complex. The piece begins at a very slow tempo (about $30 \mathrm{MM}$ per crochet-note beat), and the internal form of the 32-beat drumming pattern is an 'antecedent-consequent' pattern in the configuration of four 8-beat phrases in the pattern $A, A, A, B$ (illustrated in Figure 6). The 8beat drum pattern $A$ comprises two shorter 4-beat phrases $(a, b)$ played consecutively and coinciding on beat 8 with the high-pitched tetawak gong tone. This drumming pattern $A$ is repeated twice more to coincide with the high-pitched gong tone on beats 16, and 24. The final 8-beat pattern in this piece (noted as pattern $B$ ) serves as the cadential rhythmic pattern, which is derived from rhythmic figures found in the last 4 beats of the main pattern $A$. The 8-beat pattern $B$ ends on beat 32 of the periodic gong unit simultaneously with the sounding of the low-pitched tetawak gong tone.

Figure 6. The resultant drum rhythmic patterns for the piece "Sedayung Mak Yong".

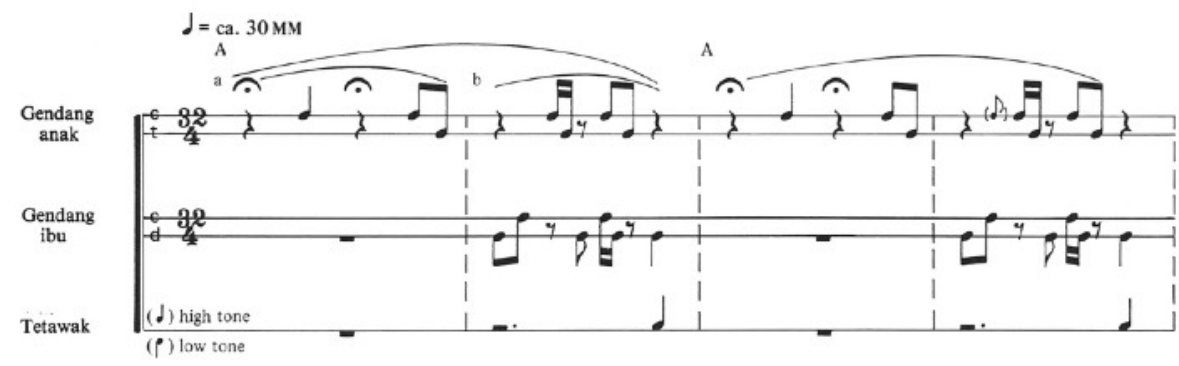

A s noted earlier, the 32beat periodic unit (or gong $\mathrm{u} \quad \mathrm{n}$ i $\mathrm{t}$ ) consistently guides the occurrence and sequence of the vo c a 1 a n d chorus parts,

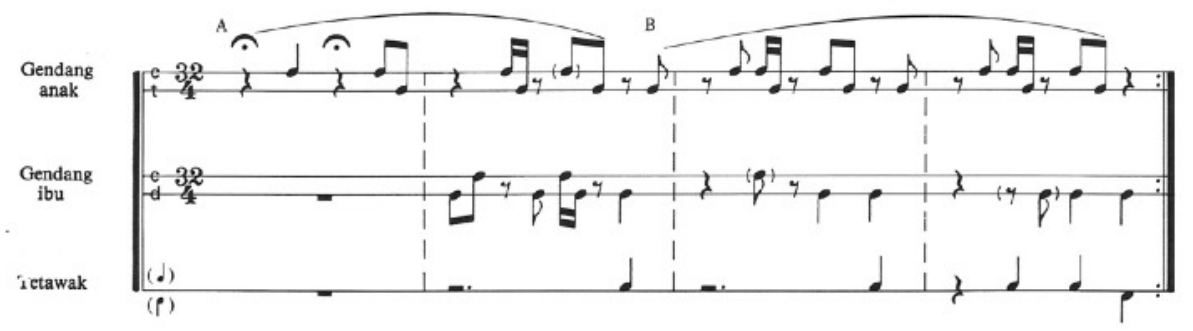
Key to the symbols:

c = mnemonic "chak" $\curvearrowright=$ a held or sustained beat longer in duration than the other $\mathrm{t}=$ mnemonic "ting" $\quad \therefore=$ beats, played at the discretion of the performers.

$\mathrm{d}=$ mnemonic "duh" $\quad$ (d) $=$ drum stroke may or may not be played. with the solo $\mathrm{V}$ o performing throughout one complete gong unit, then the chorus entering on the final beat of the soloist's part (and gong unit) and continuing to sing throughout the next successive gong unit. The solo and chorus parts overlap by one or two beats at the end of one gong unit and the beginning of the next successive gong unit, thus providing a sense of continuity in the melodic lines of the song and perhaps an attempt to obscure the rigid structure of the gong unit. All pieces 
entitled 'sedayung", and in fact, most pieces in the musical repertory of the mak yong, exhibit the stylistic trait of 'overlapping vocal parts' at the end of one gong unit and the beginning of the next. ${ }^{7}$

In the songs under discussion, the improvised lyrics or textual lines sung by the solo singer are usually fragmented into four phrases, each phrase sung in the time of a quarter-segment of a gong unit and ending with an open syllable coinciding with a gong tone. Typical open syllables are "-ma," "-na", "-weh," or "$g a$ " and so on, which often incorporate a lengthy melisma (many notes sung on a single syllable of text). The lyrics by the chorus (the jong dondang) are limited to the word 'dondang' (lit., 'lullaby'; in the local dialect pronounced as 'don-dae', thus maintaining the open syllable at the end of a textual/melodic phrase). This 2-syllable word is repeated over and over by each chorus member with her own melodic and rhythmic variations. These syllables and words, too, are sung within the context and structure of the given gong unit of the song. Whenever the gong unit and the drumming patterns accompany the chorus part in the 'Sedayung Mak Yong', the periodic unit is the dominant element providing the tempo, a framework for the antecedent-consequent structure of the drum part, and all melodic lines sung by the chorus. Any improvisational interplay by the drummers also takes place within the given 32-beat gong unit.

However, during the solo vocal part by the Mak Yong character, a more flexible and discursive relationship usually takes place between the vocal solo lines, the drumming part and the gong unit. These moments in the song may be seen in the long-held, sustained beats shown in Figure 7 shown near the end of this article — beats 3,11,17 and 19 - a time when the lyrics and the vocal melodic line become dominant and take over the timing and duration of main beats in the gong unit and in the drum patterns. At this point in the music, the gong unit and the melodic content negotiate aspects such as timing, tempo and occurrence of specific drum timbres, and a compromise is achieved in the conversation and performance between these parts in the musical texture.

As seen in the score of Figure 7, the 'sustained' sign (fermata) indicates a beat longer in duration than all other beats which, in turn, delays the beat to follow. During the sustained beats the drummers and the gong player follow the lead taken by the singer, as they wait for her to complete the melismatic melodic phrase and lyrics before they resume playing the drum pattern at the tempo and timing originally established. The drummers' performance experience with the solo singer's style and with the kinds of formulaic melodic ornaments commonly used in the vocal line serve to cue the drummers to the fact that the 'sustained' beat is concluding and the gendang anak player resumes his drum pattern to establish the original tempo of the song.

Pointing to some examples that demonstrate this phenomenon, in Figure 7 we note that on the slightly sustained beat 3 the lyric ' $[k e]$-lu-ar' is sung using a melodic line featuring fast running notes incorporating a grace note and a musical turn that then lead to the original tempo on beat 4. Another example may be seen on the sustained beat 11 on which the singer executes the lyrics ' $[a]$-bang weh' using a drawn-out portamento singing style in which the voice glides from pitch to pitch in the intended melodic line in a tempo determined by the singer at the time of performance, finally ending on beat 12 where the original tempo resumes in the vocal, drum and gong unit parts. A final example occurs on beats 17-19 when the voice rests and the rebab takes up the melodic line with an extremely fast trill (notes $B$ flat to $A$ ) and concludes with a descending melodic contour emphasizing an augmented $2^{\text {nd }}$ interval (notes $A$ to $G$-flat) on beat 19 . Here, too, the rebab player sets the tempo at his discretion, thus drawing out the time of beats 17-19 for several seconds. The rebab part continues with much repetition of the augmented 2nd interval throughout beat 19, and it is only with the accented pitch (G-flat) on beat 20 that the drummers resume their original tempo and continue with the intended rhythmic pattern. In these examples, the iso-period (or gong unit) itself is interrupted for several seconds at a time; in effect, the gong unit must wait for the voice or rebab to complete their parts, in effect negotiating with the musical content (the melody) which is the pre-dominant factor in the musical texture at the given beats in the examples noted above. 
At the specific 'sustained' beats in the gong unit as noted above, the singer demonstrates her vocal skill in improvising the sung lyrics, usually using a highly melismatic and ornamented melodic line, and the rebab player may follow suit as on the beats 17 and 19 in Figure 7. On the sustained beats of the piece 'Sedayung Mak Yong', the lyrics and melody become the dominant factors, and the drumming pattern as well as the gong unit fall to the background of the musical texture, so to speak, for the duration of those particular beats in the overall periodic unit. It is at these moments in the song that the creative skill of the performer is allowed to dictate the occurrence of tempo, beats and timbres in the gong unit itself.

Vocal and Instrumental Melody The solo vocal lines and the rebab bowed-lute parts weave complex and highly ornamented melodies in most pieces of the mak yong repertory, which is exemplified in the piece 'Sedayung Mak Yong'. In this and other pieces in the musical repertory, the voice and rebab perform in a heterophonic relationship to each other as they play related yet independent parts. ${ }^{6}$ In a mak yong piece, the voice and rebab simultaneously, or alternately, perform the same basic melody but with individual variations of it, composed at the discretion of the performing artists. Heterophonic texture also occurs in mak yong music among the various parts in the chorus, the jong dondang, in which the text 'don-dae' is sung and repeated over and over by all singers following a basic melodic contour (not necessarily a specific melodic line) but with each singer singing her own melodic and rhythmic variations within the given contour and lyrics (a transcription of heterophonic parts by the chorus is given in Matusky 1994). Interestingly, the performance of a sung melody by a solo voice accompanied by a lute (or other instrument), or by a group such as a chorus singing the same melody in heterophonic style, is found in musics throughout the Islamic world from north Africa to parts of the Middle East, and also in the mak yong of Malaysia where Islam is the dominant religion among Malay and other peoples. It should also be noted, however, that while Middle Eastern influence may be seen here, the heterophonic singing by a group of singers is also found among some indigenous groups in Sarawak and perhaps in other parts of Southeast Asia as well. ${ }^{8}$

Considering tonal characteristics of melody in the mak yong repertory, core sets of pitches comprising scales (or pitch-class sets) for various pieces may consist of 4,5 , or 6 specific pitches (or pitch classes). The piece 'Sedayung Mak Yong', for example, shows a seemingly limited tonal vocabulary utilizing a basic core of 6 pitches within the range of the interval of a fifth (pitches $D$ to $G$ in the transcription shown as Figure 7, the $3^{\text {rd }}$ gong unit of the piece). During performance of the melodic line some of the core pitches are sharpened or flattened by a semitone or less, thus incorporating microtones as well as diminished and augmented intervals to color the melodic line and increase the richness of the tonal vocabulary.

As shown in Figure 7 included near the end of this article, on beats 9 through 16 the $1^{\text {st }}$ scale degree (pitch $G$ in the transcription) is flatted and an augmented $2^{\text {nd }}$ interval (pitches $A$ to $G$-flat) is introduced by the singer and repeated in the melody (either by the singer or the rebab player) at various times in the remainder of the gong unit. There are times when the vocal melodic line is at rest or is static (see Figure 7 below, beats 17-24), and at these times the rebab player takes the opportunity to execute a highly volatile melodic line featuring many fast-running notes, triplets, and turns, thus continuing to color the melodic line with conjunct motion along with the augmented $2^{\text {nd }}$ interval. In spite of the rather limited melodic range noted here (the interval of a $5^{\text {th }}$ ), the dynamism and richness in the melodic parts of the mak yong piece are displayed and maintained through the use of chromatic tonal vocabulary along with a high degree of melodic ornamentation.

The singing style of village mak yong singers, especially throughout the $20^{\text {th }}$ century, was characterized by the use of a strident tense voice quality and some degree of nasalization. In the days of no electronic technology in the village that would enable the use of microphones and voice amplification, the strident voice quality was able to project the vocal sound naturally, including the high degree of vocal ornamentation used by the singer. It is possible that this style continues to this day in some village venues, and it is also possible that this style is changing in an urban, indoor setting with the use of microphones and other forms of amplification. 
Many of the vocal ornaments emphasizing microtonal intervals are not unlike those ornaments heard in vocal styles from the Islamic Middle East and frequently found in the vocalization of religious texts. One of the common melodic ornaments vocalized by mak yong singers is the grace note (acciaccatura) in the form of a whole step or a semitone before the downbeat of a given pitch (see Figure 7 below, beats 3-4, $6-7,10,13,15$ ). Another vocal ornament is a tremulo (or wobble) which is an excessive wavering of a given pitch that can be quite wide, sometimes encompassing an interval of a $2^{\text {nd }}$ or $3^{\text {rd }}$. The glottal stop is also a common vocal ornament often occurring on the syllables é or ' $g a$ ' (see Figure 7, beats 31a-32a), and the portamento (or slide) in which the voice glides from one pitch to another is often found in a melismatic passage (see Figure 7, melisma on beats 11-12 on the syllable 'weh'). Other melodic ornaments in the vocal and rebab parts include the downward release of a pitch as shown in Figure 7 (beat 14), the trill (beat 6) and the turn (beats $3,18-19$ ).

As the singer weaves her highly ornamented melodic lines, the melody and text become the dominant aspects in the music, overriding the tempo set by the drummed patterns, as well as the occurrence of drum timbres on certain beats and the running beats contained in the periodic unit (the gong unit). In effect, on certain 'sustained' beats (as noted above) the drummed patterns and the periodic unit halt or stand still for an indeterminant length of time, in a seemingly interstitial place in time, where neither the gong unit nor the resultant rhythmic patterns have control in the musical texture. The drummed parts and the gong unit must wait for the singer to complete a line of lyrics or a melismatic melodic line, and only then can the periodic time unit once again proceed to mark out specific beats as played by the high- and low-pitch gong tones. Here we see extreme negotiation in which the melody is clearly dominant in the musical texture during the few beats in the gong unit devoted to pure melody rendered by the human voice or the rebab (an instrument possibly seen as an extension of the human voice).

\section{Conclusion}

As noted in the foregoing discussion, the melodies found in the mak yong musical repertory exhibit a limited melodic range, and yet a high degree of melodic ornamentation (trills, turns, grace notes, portamento style, downward release of pitch, glottal stop), and the use of chromatic and micro tones that enrich a basic core scalar pattern of six pitches as the tonal vocabulary. In addition, an important musical feature is the consistent use of heterophonic texture within the context of a chorus of singers, and also in the context of a soloist accompanied by a bowed-lute instrument. It is clear that the melodic style of mak yong pieces, such as the 'Sedayung Mak Yong' discussed above, exhibits a complex melodic content in most, if not all, pieces in the repertory.

As this discussion has attempted to show, the complex melodic content is only one part of a stratified musical texture that also includes drummed resultant rhythmic patterns and the tones of the gong unit. The percussive drum patterns accompany the vocal and rebab melodies, and they also establish a perceived running beat in the music with a given tempo as two players strike their respective drums in an interlocking style to produce the resultant patterns. These patterns usually exhibit a particular internal structure using specific mnemonic timbres to produce antecedent-consequent patterns, or simply iterative patterns punctuated by specific cadential phrases. In all cases, the resultant and cadential patterns by the drummers occur within the context of the iso-periodic formal structure as rendered by the large, bronze knobbed gongs in the music ensemble.

The iso-periodic units (or gong units) in all mak yong pieces (as in some other traditional Malay music in which knobbed gongs are found in the music ensemble) are cyclic and binary in nature and in structure. The gong unit in a given piece is usually unchanging in formal structure and is repeated over and over in succession until a piece comes to an end. The gong unit features hierarchical beats, with the beat of greatest importance and stress occurring at the end of a given gong unit. The gong unit provides a given piece with a specific musical formal structure that gives unity, sense and logic to the musical sound. While cyclic gong units tend to be highly rigid and unchanging in their form, they can also be discursive in nature and become somewhat flexible at specific times in the music. It is at these discursive times in the musical soundscape that the gong unit takes a less dominant role in determining music form in 
relation to other parts of the musical texture, and in the case of the mak yong it is usually the sung vocal melody or the rebab melody that pre-dominates at these discursive times. The iso-period and the melodic parts, in effect, negotiate the elements of tempo, timing, occurrence of percussive beats, and the running beats in the gong unit. Negotiation is necessary so that the melodic part along with the lyrics may project the dramatic expression at given moments in a story. The gong unit assumes a subsidiary role at these discursive times, and yet it still provides a framework in which given song lyrics may be sung by the singer along with beautiful melodies. When complex musical content and periodicity negotiate, the result is a dynamic musical style that underpins and supports the dramatic aspects of this traditional Malay theatre form.

\section{Endnotes}

${ }^{1}$ The historical and dramatic aspects of the mak yong have been discussed in great detail by Ghulam Sarwar Yousof in 'The Kelantan Mak yong Dance Theatre: A Study of Performance Practice'. PhD Dissertation, University of Hawaii, 1976.

2 This observation is based on my conversations during field research with Malay villagers in Kelantan, Narathiwat and Patani (southern Thailand) in 1975-76, during my residence in Yala, Thailand in 1982, and most recently during my residence in Malaysia in the early decades of the $21^{\text {st }}$ century. Since the early 1990s and the dominance of the Islamic-based political party in the state of Kelantan, the performances of mak yong and other traditional arts in Kelantan have been banned as being un-Islamic.

${ }^{3}$ In an urban setting the proscenium stage or any other raised and partially walled-in platform area may also be used for performances.

${ }^{4}$ Comprehensive lists of mak yong pieces are noted in Ghulam Sarwar, 1976: 123-129, and in Malm 1974: 21.

${ }^{5}$ See further details of these and other instruments in Matusky 1980 and 1994.

6 'Heterophony' is a type of polyphonic texture in which there is simultaneous use of modified versions of the same melody performed by two or more performers. There may be variations in pitch as well as in rhythm of the basic melody.

${ }^{7}$ The solo singer usually ends a given song, and at the end of her final sung text and conclusion of the gong unit in which that sung text occurs a known, standard cadential drum rhythmic pattern and gong unit is played upon cue from the singer (a nod of the head, a bodily movement or other cue given by the singer as the final beat of the gong unit approaches. This cue, then, directs the drummers and gong player to conclude the piece with the cadence pattern appropriate for the given piece).

${ }^{8}$ Among the Kajang-Sekapan and other Kajang groups in the Upper Rejang River region of Belaga District, Sarawak, the singing of narratives and other songs are often sung by a soloist alternating with a chorus that sings a refrain usually in heterophonic style. See further Matusky 2012:303, and 2004:274, and Sarawak Museum Journal, XXXVI: 185-229. 

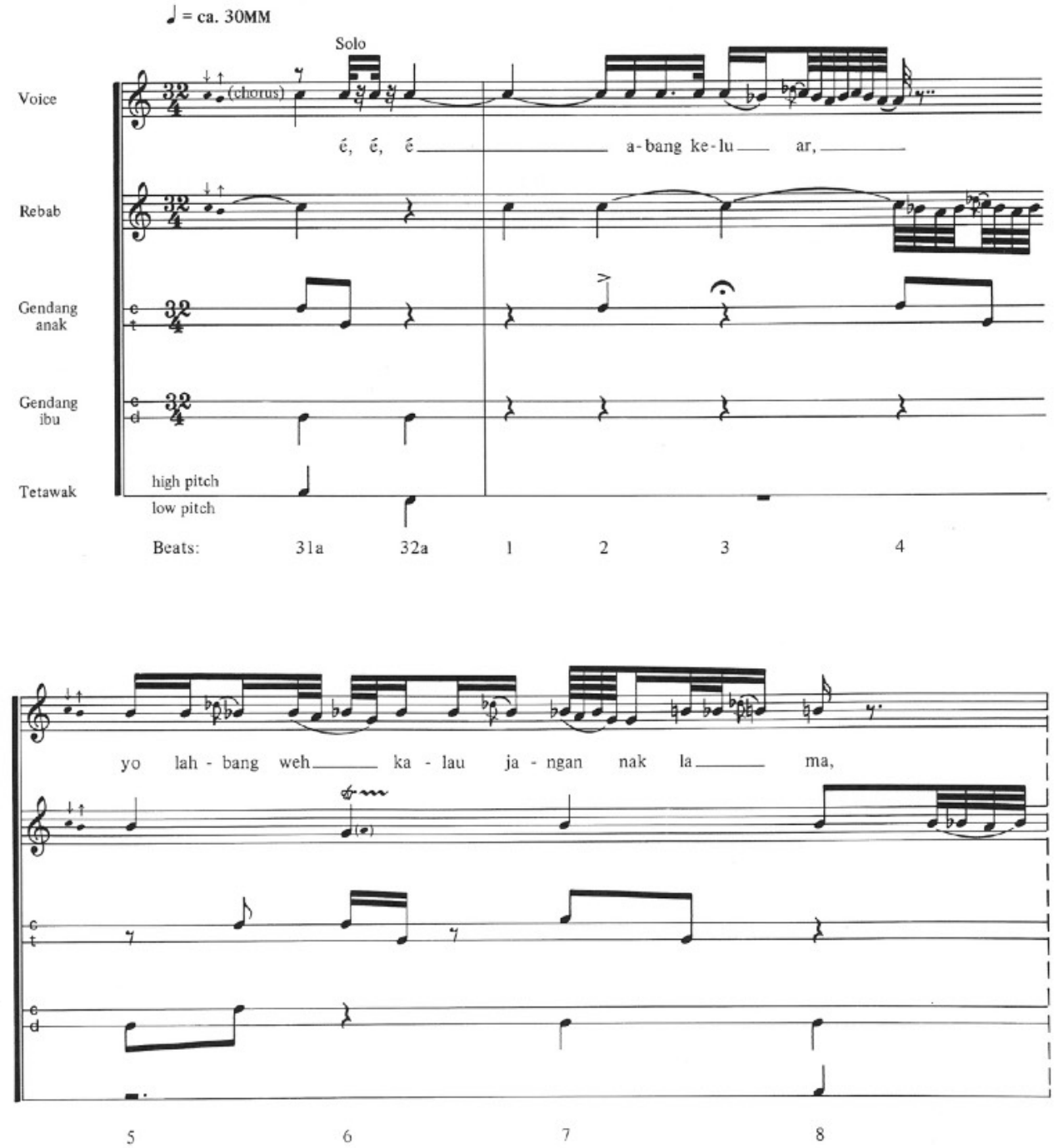

This piece was recorded by Dr. Ghulam-Sarwar Yousof in Kota Bharu, Kelantan, in 1975 and transcribed by the present author. 

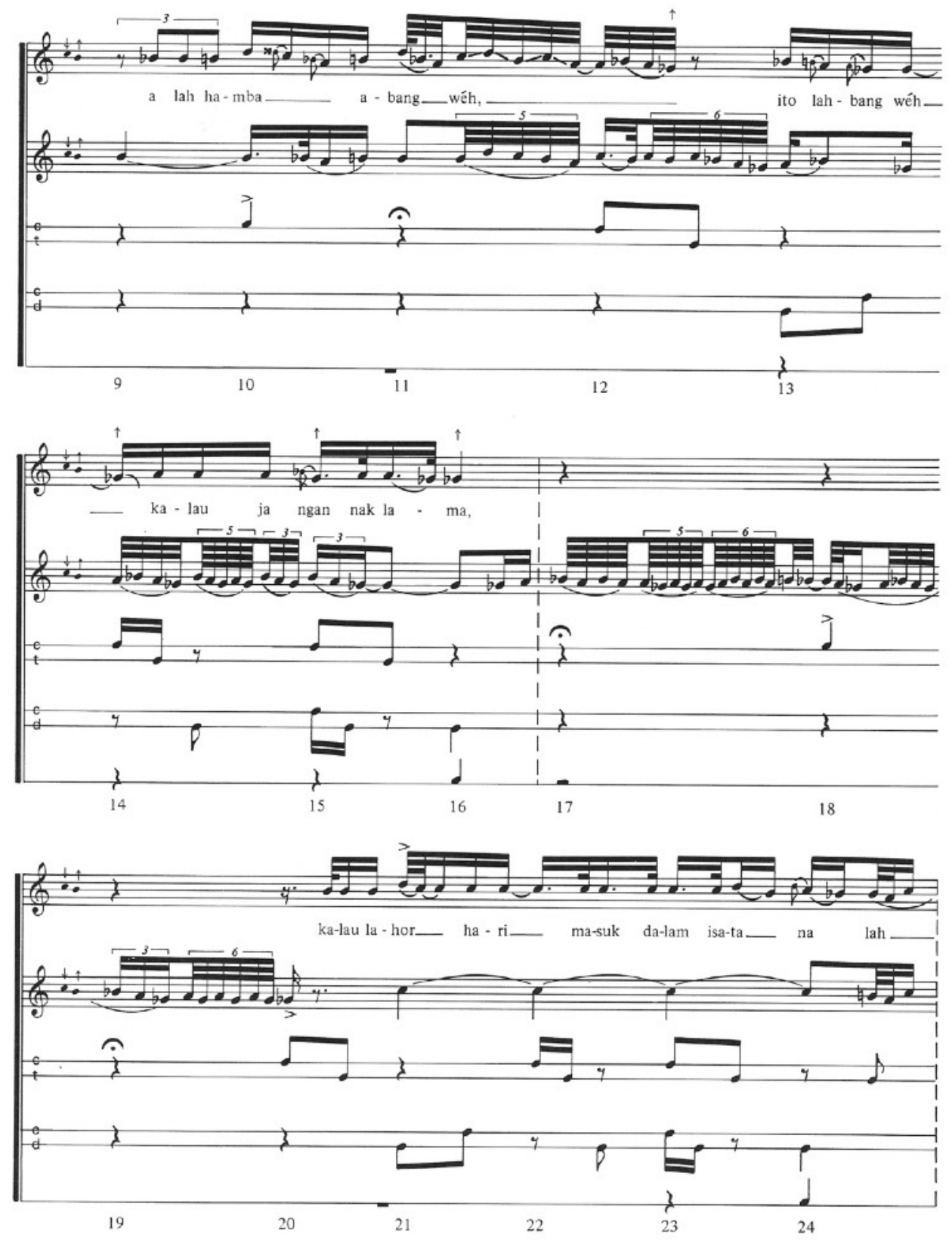

continued 

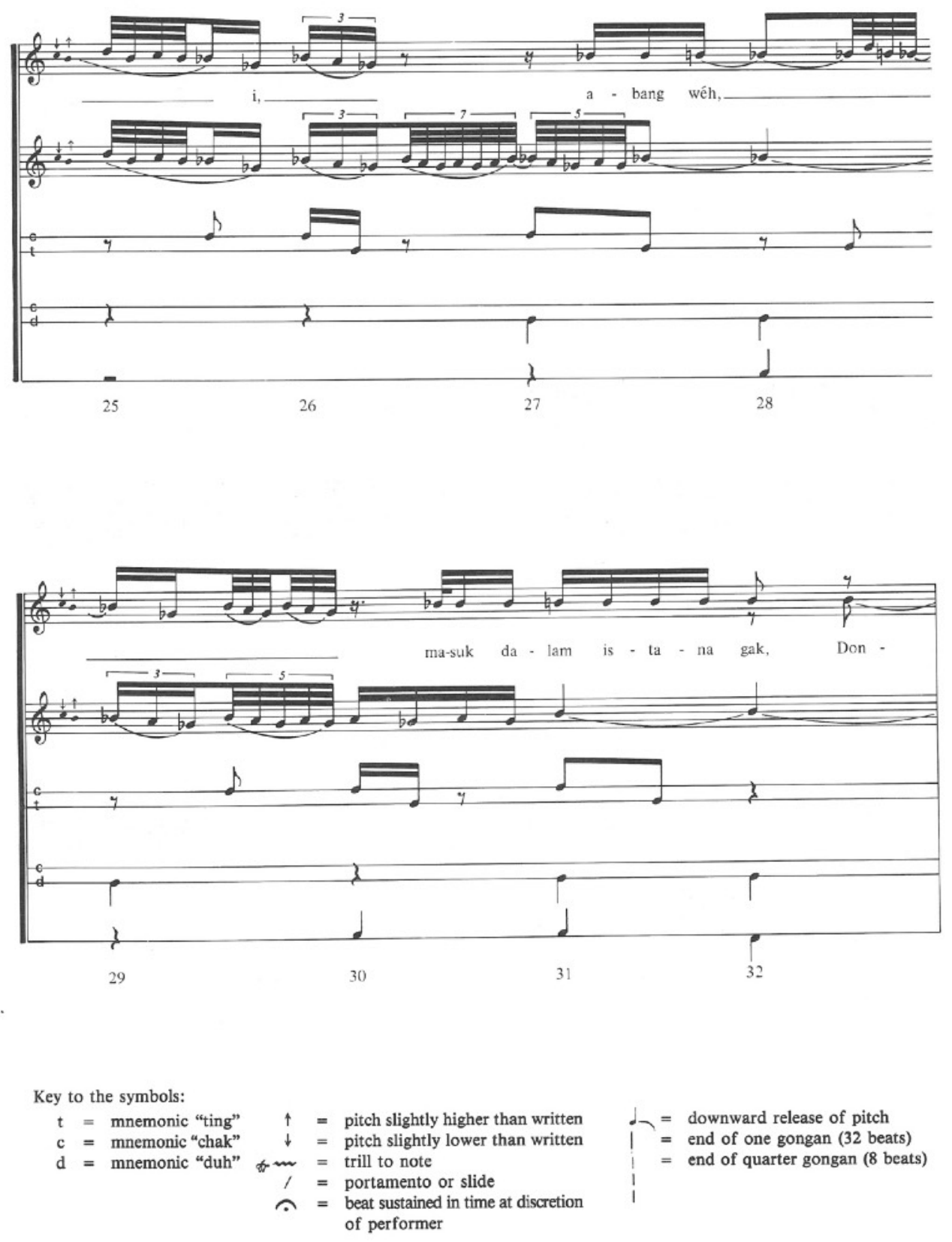

Figure 7. 'Sedayung Mak Yong' (third gong unit only), recorded by Ghulam-Sarwar Yousof in Kota Bharu, Kelantan in 1975, and transcribed by P. Matusky.

\section{Select Bibliography}

Becker, Judith. (1979). "Time and Tune in Java." The Imagination of Reality: Essays in Southeast Asian Coherence Systems. Norwood, New Jersey: ABLEX Publishing Co., 204-08.

Farmer, George. (1926). A History of Arabian Music. London: Luzac. 
Fernando, Jacqueline Sunetra. (1996). 'Lagu Menghadap Rebab in the Mak yong Theatre of Kelantan and South Thailand: an interpretive musical analysis'. MA Thesis, Kuala Lumpur: University of Malaya.

Ghulam Sarwar Yousof. (1976). The Kelantan Mak yong Dance Theatre, A Study of Performance Structure. PhD Dissertation, University of Hawai'i at Manoa. Ann Arbor: University Microfilms.

. (1976). 'Revival of an Ancient Tradition: The Mak yong Dance-Theatre of Malaysia,' In Culture Learning Institute Report. Honolulu, Hawaii: East-West Center, 4 (3): 3-5,11,13.

(1982). 'Mak yong: The Ancient Malay Dance-Theatre,' In Asian Studies, Manila: University of the Philippines, 20: 108-121.

. (1992). Panggung Semar, Aspects of Traditional Malay Theatre. Kuala Lumpur: Tempo Publishing (M) Sdn Bhd.

Malm, William P. (1977, 1967). Music Cultures of The Pacific, the Near East, and Asia. Englewood Cliffs, New Jersey: Prentice-Hall, Inc.

. (1969). 'Music of the Ma'Yong', In Tenggara 5:114-120.

(1971). 'Malaysian Ma'Yong Theatre,' In Drama Review. 15 (3):108-114.

(1974). 'Music in Kelantan, Malaysia and Some of Its Cultural Implications', In Studies in Malaysian Oral and Musical Traditions. Michigan Papers on South and Southeast Asia, 8. Ann Arbor: University of Michigan Center for South and Southeast Asian Studies.

Matusky, Patricia. (1980). Music in the Malay Shadow Puppet Theater. PhD Dissertation, University of Michigan. Ann Arbor: University Microfilms.

. (1982). 'Musical Instruments and Musicians of the Malay Shadow Puppet Theater', In Journal of the American Musical Instrument Society, 8:50-56.

(1994). Music of the Mak yong Theater: A Fusion of Southeast Asian Malay and Middle Eastern Islamic Elements', In To The Four Corners: A Festschrift in Honor of Rose Brandel. Ellen C. Leichtman, Ed., Warren, Michigan: Harmonie Park Press.

and Tan, Sooi Beng. (1997, 2012). Muzik Malaysia, Tradisi Klasik, Rakyat dan Sinkretik. Kuala Lumpur: Universiti Malaya Press.

Mohd. Affandi Ismail. (1974-74). 'Mak yong-Sebuah Tinjauan dari Sudut Persembahan'. MA Thesis, University of Malaya. $\overline{19: 363-83 .}$

(1975) 'Perkembangan Mak yong Sebagai satu Seni Teater Tradisional." Dewan Bahasa

Sachs, Curt. (1940). The History of Musical Instruments. New York: W.W. Norton \& Company.

Tenzer, Michael. Ed. (2006). Analytical Studies in World Music. Oxford: Oxford University Press. 